\title{
VLBI Image Deconvolution Based on Generalized Nonlinear In- formation Methods
}

\author{
A. T. Bajkova
}

Institute of Applied Astronomy, St. Petersburg, 197110, Russia

\begin{abstract}
A number of nonlinear image deconvolution procedures based on extremizing generalized Shannon entropy, Kullback-Leibler cross-entropy and Renyi information measures are suggested for VLBI mapping with minimal nonlinear distortions. A new approach to phaseless mapping urgent in the case of unreliable closure phases is proposed.
\end{abstract}

\section{Introduction}

Maximum entropy method (MEM) and other information methods are widely used for image reconstruction from incomplete and noisy Fourier data. The "generalization" assumes search for a solution over the space of complex functions (Bajkova 1992). Such an approach allows, first, to map coherent (complex) radio sources (Bajkova 1995, Bajkova et al. 1995) and, secondly, to decrease nonlinear image distortions caused by measurement errors. All the procedures are contained in "IMAGE" program package developed in IAA RAS and were applied to mapping compact extragalactic radio sources (Frieden \& Bajkova 1995).

\section{Generalized Maximum Entropy Method}

Let us consider a complex signal $u_{m l}=r_{m l}+j q_{m l}, j=\sqrt{-1}$, where both $r_{m l}$ and $q_{m l}$ are real bipolar signals represented as follows

$$
r_{m l}=x_{m l}-y_{m l}, \quad q_{m l}=z_{m l}-v_{m l},
$$

where $x_{m l} \geq 0, y_{m l} \geq 0, z_{m l} \geq 0, v_{m l} \geq 0$.

The minimized entropic functional for estimation of the complex signal is written as (Bajkova 1992):

$$
\min \sum_{m} \sum_{l} x_{m l} \ln \left(\alpha x_{m l}\right)+y_{m l} \ln \left(\alpha y_{m l}\right)+z_{m l} \ln \left(\alpha z_{m l}\right)+v_{m l} \ln \left(\alpha v_{m l}\right)
$$

with inserted a positive parameter $\alpha$ responsible for not overlapping positive and negative parts of the sequences $r_{m l}$ and $q_{m l}$.

\section{Generalized Kullback-Leibler Method}

Generalized Kullback-Leibler method (Bajkova 1995) assumes prior knowledge of bias non-negative functions $a_{m l}, b_{m l}, c_{m l}, d_{m l}$ (it may be "dirty" image) corresponding to the signals $x_{m l}, y_{m l}, z_{m l}, v_{m l}$ respectively. Then functional (2) is modified as

$$
\begin{aligned}
& \min \sum_{m} \sum_{l} x_{m l} \ln \left(\alpha x_{m l} / a_{m l}\right)+y_{m l} \ln \left(\alpha y_{m l} / b_{m l}\right) \\
& \left.+z_{m l} \ln \left(\alpha z_{m l} / c_{m l}\right)+v_{m l} \ln \left(\alpha v_{m l} / d_{m l}\right)\right)
\end{aligned}
$$




\section{Generalized Minimum Renyi Information Method}

Representing the sought for sequences also in the form (1) we can write generalized Renyi functional as follows (Bajkova et al. 1995)

$$
\min \sum_{m} \sum_{l} x_{m l}^{s} a_{m l}^{1-s}+y_{m l}^{s} b_{m l}^{1-s}+z_{m l}^{s} c_{m l}^{1-s}+v_{m l}^{s} d_{m l}^{1-s},
$$

where $a_{m l}, b_{m l}, c_{m l}, d_{m l}$ are corresponding reference signals.

It is established that the quality of image reconstruction depends on the choice of parameter $s$ in the functional. When $s=2$ we have a conventional minimum intensity criterium. Experimentally established that when $s$ approaches 1 we obtain a criterium with more strong nonlinear (extrapolation) features approaching maximum entropy method.

Because of nonlinearity nature of information methods there is a problem of nonlinear image distortions caused by errors in data (Frieden \& Bajkova 1994). We consider only additive errors. As experiments show the generalized algorithms ensure much less distortions than the classical ones. This phenomenon is explained by the fact that to noisy data in general a complex solution corresponds.

\section{Phaseless Mapping and the "IMAGE" program package}

In the case of unreliable closure phases using phaseless image deconvolution algorithms can be of great interest. There exists the well known Fienup's iteration algorithm. But usually because of UV-coverage is not complete, Fienup's algorithm works badly. We propose first to reconstruct with maximally high quality the visibility amplitude using the best deconvolution algorithm and only then to use Fienup's algorithm.

"IMAGE" program package elaborated in IAA RAS in framework of "QUASAR" VLBI project is intended for VLBI mapping and investigation of new image reconstruction procedures. This package can work in combination with "CALTECH" programs. Maps of compact extragalactic sources obtained using the generalized and phaseless procedures are shown in Frieden \& Bajkova (1995). We used geodetic VLBI data of GSFC.

\section{References}

Bajkova, A. T. 1992. Astron. E\& Astroph. Tr., 1, 313-320.

Bajkova, A. T. 1995. Izv. Vuzov. Radiofizika, 38, 1267-1277.

Bajkova, A. T., Pyatunina, T. B. \& Finkelstein, A. M. 1995. Proc. IAA RAS , 1, 22-63.

Frieden, B. R., \& Bajkova, A. T. 1994. Appl. Opt., 33, 219-226.

Frieden, B. R., \& Bajkova, A. T. 1995. Appl. Opt., 34, 4086-4093. 